\title{
Circ-0036602 Acts As a Sponge of MiR-34a-5p and MiR-431-5p to Promote Cervical Cancer Proliferation and Invasion
}

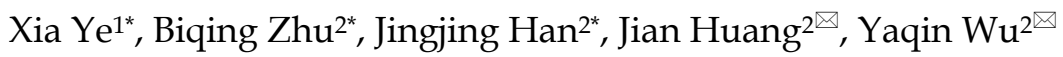 \\ 1. Department of Radiation Oncology, The First People's Hospital of Nantong, The Second Affiliated Hospital of Nantong University, Nantong 226001, China. \\ 2. Department of Radiation Oncology, Jiangsu Cancer Hospital, Jiangsu Institute of Cancer Research, The Affiliated Cancer Hospital of Nanjing Medical \\ University, Nanjing 210009, China. \\ * These authors contributed equally to this work. \\ $\triangle$ Corresponding authors: Yaqin Wu; Jian Huang. No. 42, Baiziting Road, Nanjing, 210009,China. Tel: +86 13770792530 ; +86 1891 3985 006; Email: \\ wyq-087@163.com; huangjian7107@163.com.
}

() The author(s). This is an open access article distributed under the terms of the Creative Commons Attribution License (https://creativecommons.org/licenses/by/4.0/). See http://ivyspring.com/terms for full terms and conditions.

Received: 2021.05.07; Accepted: 2021.09.29; Published: 2022.01.11

\begin{abstract}
Background: Cervical cancer (CC) is one of the most common female malignancies worldwide. An increasing body of evidence suggests that circular RNAs (circRNAs) participate in the pathogenesis of various cancers, including CC. However, the expression profile and underlying molecular mechanisms remain largely unknown.

Methods: In this study, high throughput sequencing was applied to identify circRNA in HPV-16 positive CC tissues. Quantitative real-time PCR (qRT-PCR) was performed to validate the expression in $C C$ tissues and cell lines. RNase $R$ treatment, gel-electrophoresis, and RNA fluorescent in situ hybridization (FISH) were used to characterize the circRNAs. Subsequently, the Cell Counting Kit-8 assay (CCK8), transwell and wound healing assays were performed to assess circRNA function. Meanwhile, dual-luciferase reporter and western blot were used to clarify the associated molecular mechanisms.

Results: Circ0036602 was upregulated in HPV-16 positive CC and correlated with a poor prognosis. Moreover, circ0036602 expression significantly correlated with the clinicopathologic characteristics. Knockdown of circ0036602 inhibited CC cell proliferation, migration, and invasion. Further studies showed that circ0036602 could bind to miR-34-5p and miR-431-5p to regulate the expression of the target gene HMGB1.

Conclusions: Taken together, our findings suggest that circ0036602 is a tumor-promoting circRNA that promotes CC cells by sponging miR-34-5p and miR-431-5p to regulate HMGB1. Circ0036602 has huge prospects as a potential therapeutic target for CC patients.
\end{abstract}

Key words: Cervical cancer; circ0036602; circRNA; HPV; invasion and metastasis

\section{Introduction}

Cervical cancer (CC) is the most common gynecological cancer and has become the second leading cause of cancer-related deaths among women $[1,2]$. There are about 570,000 new cases reported and 311,000 deaths annually [1]. Although HPV vaccines have shown promising results over the past decades, they are prophylactic and have no benefit on active disease infection [3, 4]. In addition, multivalent vaccines do not cover all types of HPV known to cause CC [5]. It has been reported that more than $50 \%$ of cervical cancer patients are infected with HPV-16, documented as the predominant type in CC $[3,6]$. 
Interestingly, recent studies have demonstrated that the carcinogenicity of HPV-16 is associated with the highly conserved E7 protein that can promote the occurrence and development of HPV-16 positive cervical cancer [7]. Accordingly, it is of great significance to investigate the molecular mechanisms, and explore prognostic and therapeutic targets for CC.

CircularRNAs (CircRNAs) are a class of single-stranded RNA characterized by a covalently closed loop short of the $5^{\prime}$ caps and $3^{\prime}$ poly-A tails, making them more stable than linear RNAs [8-10]. They are endogenous, conserved, and abundant [11]. CircRNAs have long been regarded as splicing byproducts with little functional potential [12]. In recent years, emerging evidence has revealed that circRNA can exert functions as microRNAs (miRNAs) sponge [13], competing for miRNA binding, or gene transcription modulators to regulate target genes expression [14] and interact with RNA-binding proteins $[15,16]$. Moreover, many circRNAs have been demonstrated to be involved in various cancer-related biological processes, including cell metastasis, apoptosis and proliferation in CC $[17,18]$. For example, circFNDC3B inhibits colorectal cancer metastasis, invasion, and angiogenesis by sponging miR-937-5p to derepress TIMP3 expression [19]. Moreover, circRNA NFATC3 may act as a miR-9-5p sponge and regulate the SDC2/NF-kB signaling pathway in CC [20]. Besides, circ0067934 upregulation in CC reportedly promotes disease progression via the miR-545/EIF3C axis [21]. These studies indicated that circRNA could be used as candidate biomarkers and therapeutic targets. With the development of high-throughput sequencing and advanced bioinformatics, an increasing number of circRNAs has been discovered in mammalian cell lines and across various species; however, their regulatory roles and clinicopathological relevance in CC are not well understood and need further exploration.

This study found that circ0036602 was upregulated in HPV-16 positive CC, deprived of exons (exons 5 10) of the ZNF592 gene and negatively associated with differentiation, $\mathrm{N}$ stage, and poor prognosis. Furthermore, our data demonstrated that circ0036602 silencing inhibited CC cell proliferation and invasion by acting as a sponge of miR-34a-5p and miR-431-5p. Overall, our findings suggest that circ0036602 is an oncogene and has huge prospects as a novel biomarker and a therapeutic target for CC.

\section{Methods}

\section{Human species}

In total, $30 \mathrm{HPV}-16$ positive $\mathrm{CC}$ tissues and matched HPV-16 negative CC tissues were harvested at the Jiangsu cancer hospital from June 2018 to July 2020 and stored in liquid nitrogen for later use. None of these patients received chemotherapy and radiotherapy prior to specimen collection. Written informed consent was obtained from all participants, and the study was approved by the Ethics Committee of The Affiliated Cancer Hospital of Nanjing Medical University. The study protocol was performed in accordance with the ethical standards of the Declaration of Helsinki. All collected tissues were examined by experienced pathologists.

\section{Cell Culture}

CC cell lines Caski, Siha, C33A and normal cervical epithelial Hacat, purchased from American Type Culture Collection (ATCC), were cultured in Roswell Park Memorial Institute (RPMI) 1640 medium with $10 \%$ fetal bovine serum (FBS) and 1\% penicillin at $37^{\circ} \mathrm{C}$ with $5 \%$ carbon dioxide.

\section{Cell Transfection}

Small interfering RNAs (siRNA) targeting circ0036602, and siRNA control were designed by Ribobio (Guangzhou, China). Caski and Siha cells were cultured in a 96-well plate, incubated overnight at $37^{\circ} \mathrm{C}$, and transfected when the cells were grown to $50 \sim 60 \%$ confluence. After $48 \mathrm{~h}$ transfection, the cells were harvested, and the transfection efficiency was assessed by quantitative real-time PCR (qRT-PCR) analysis. Lipofectamine 2000 was used for transient transfection according to the manufacturer's protocol.

\section{Genomic DNA, RNA extraction, and quantitative real-time PCR analysis}

Genomic DNA (gDNA) was extracted from cultured cells using a Blood \& Cell Culture DNA Mini Kit (Qiagen, CA, USA), as described in the manufacturer's instructions. Total RNA was isolated from CC tissues or cell lines with Trizol reagent (Invitrogen, Life Technologies, Inc, Germany) following the standard protocol. The purity and concentration were detected with a Nanodrop2000 spectrophotometer. Complementary DNA (cDNA) was synthesized with 1 ug of total RNA using PrimeScript RT Reagent kit (Takara, Japan). Quantitative real-time PCR analysis was carried out with SYBR Green Real-time PCR Master Mix (Takara, Japan). The PCR procedure consisted of predegeneration at $95{ }^{\circ} \mathrm{C}$ for $20 \mathrm{~s}$, followed by 40 cycles of degeneration at $95{ }^{\circ} \mathrm{C}$ for $10 \mathrm{~s}$, and extension at $60{ }^{\circ} \mathrm{C}$ for $45 \mathrm{~s}$. All data were presented as mean \pm standard deviation of three independent experiments, and the relative gene expression levels were analyzed using the $2-\Delta \Delta \mathrm{Cq}$ method normalized to endogenous control genes U6 and GAPDH. The primers utilized are as follows: ZNF592 sequence: $\left(5^{\prime}->3^{\prime}\right)$ Forward 
Primer: GAATGAGAGTCCCCTCAAACCT Reverse Primer TGTAGGAGACTGGGAGTAATGTG; Circ0036602 (5'->3'): Forward Primer: CTTGTGTGCA TGAATCCGCT, Reverse Primer: CTTGTGTGC ATGAATCCGCT; miR-34a-5p: Forward Primer: GCAGTGGCAGTGTCTTAG, Reverse Primer: GGTCCAGTTTTTTTTTTTTTTTACAAC; miR-431-5p: Forward Primer: TCTTGCAGGCCGTCA; Reverse Primer: GTCCAGTTTTTTTTTTTTTTTGCAT; U6: Forward Primer: 5'-CTCGCTTCGGCAGCACA-3', Reverse Primer: 5'-AACGCTTCACGAATTTGCGT-3'; GAPDH: Forward Primer: 5'-AAGAAGGTGGTG AAGCAGGC-3', Reverse Primer: 5'-GTCAAAGGT GGAGGAGTGGG-3'.

\section{RNA isolation of nuclear and cytoplasmic fractions}

The Nuclear and cytoplasmic RNA Purification Kit (Norgen, USA) was used to extract nuclear and cytoplasmic fractions based on the manufacturer's instructions. Then, total RNA was extracted using TRIzol reagent (Invitrogen, USA) for qRT-PCR as described above.

\section{RNA fluorescent in situ hybridization (FISH)}

A specific probe targeting circ0036602 was designed and purchased from Genesee Biotech (Guangzhou, China). The cell nucleus was stained with 4,6-diamidino-2-phenylindole (DAPI) for $20 \mathrm{~min}$ and then imaged under a fluorescence microscope. The FISH experiment was performed using a fluorescence in situ hybridization kit (Ribobio, Guangzhou) to visualize the location of circ0036602 in the cell.

\section{RNase R Digestion}

RNase $\mathrm{R}$ is a $3^{\prime}$ to $5^{\prime}$ exoribonuclease that can digest circ0036602, and its linear counterpart ZNF592 mRNA. $2 \mu \mathrm{g}$ RNAs were incubated with or without RNase R (Epicentre Technologies, Madison, USA) at $37^{\circ} \mathrm{C}$ for $30 \mathrm{~min}$. Then, the relative expression levels of circ0036603 and ZNF592 mRNA were detected by qRT-PCR.

\section{Luciferase Reporter Assay}

We obtained the potential binding sites of microRNA and circ0036602 via TargetScan and Miranda, and the sequences were cloned into a psiCHECK2 vector (Promega Corporation, USA). Cell lines were seeded in 48-well plates and co-transfected with the luciferase reporter vector, and microRNA mimic or negative control with lipofectamine 2000. The relative luciferase activity was measured after 2 days of transfection by a dual-luciferase assay system (Promega Corporation, USA), according to the manufacturer's instructions.

\section{RNA immunoprecipitation (RIP) assay}

The putative relationship between circ0036602 and miR-34a-5p, miR-431-5p was further investigated by the RNA Immunoprecipitation (RIP) assay. The RIP assay was carried out using the EZ-Magna RIP kit (Millipore, USA). Briefly, cells were co-transfected with miR-34a-5p mimics or miR-431-5p mimics. The cells were harvested after $48 \mathrm{~h}$ and lysed in RIP lysis buffer. Then, the cells were incubated with proteins magnetic beads conjugated with anti-ago2 or anti-IgG antibody (Millipore, USA) at $4{ }^{\circ} \mathrm{C}$ overnight. After washing with buffer, immunoprecipitated RNA was extracted. The abundance of circ0036602 and microRNA were measured by qRT-PCR.

\section{Cell Proliferation Assay}

The Cell Counting Kit-8 was applied to determine cell viability at different time points $(0 \mathrm{~h}$, $24 \mathrm{~h}, 48 \mathrm{~h}, 72 \mathrm{~h}, 96 \mathrm{~h}, 120 \mathrm{~h}$ ), according to the manufacturer's protocol. The Caski cells in the logarithmic phase were seeded into a 96-well plate containing a volume of $100 \mu \mathrm{l}$ per well. The cells transfected with siRNA1 and siRNA2 were seeded into six replicates. $10 \mu \mathrm{l} \mathrm{CCK-8} \mathrm{(Solarbio,} \mathrm{China)} \mathrm{was}$ added to each well and incubated for $1 \mathrm{~h}$ at $37^{\circ} \mathrm{C}$. Finally, the absorbance was measured at $450 \mathrm{~nm}$ wavelength using a micro-plate, and the cell viability was calculated. All experiments were performed in triplicate.

\section{Wound healing assay}

Caski cells were divided into three groups: negative control (NC), siRNA-1, and siRNA-2 groups based on the treatment conditions. For the wound healing assay, transfected cells were seeded into 6-well plates and incubated at $37^{\circ} \mathrm{C}$ overnight until $100 \%$ confluence. A $10 \mu \mathrm{l}$ pipette tip was used to create a linear scratch and washed the detached cells with PBS. The distance of each scratch closure was subsequently observed and photographed via microscopy (100× magnification) at $0 \mathrm{~h}$ and $48 \mathrm{~h}$. Every assay was performed in triplicate.

\section{Transwell assay}

The Transwell assay was applied to assess the potential of cell migration and invasion. Transfected cells were cultured into the upper chamber containing $100 \mu 1$ serum-free medium, and the lower chamber contained $600 \mu \mathrm{l}$ complete medium harboring $10 \%$ FBS as a chemoattractant. After the cells were cultured at $37{ }^{\circ} \mathrm{C}$ for $24 \mathrm{~h}$, a cotton swab was used to gently remove cells from the upper cavity. Subsequently, the migrated cells were fixed with $4 \%$ paraformaldehyde for $20 \mathrm{~min}$ and dyed with $0.2 \%$ crystal violet. Finally, six random fields were observed under a microscope 
and photographed to measure the migrated cell count. The cell invasion assay was simultaneously performed with the above steps, except that the upper transwell chamber was pre-coated with matrigel (corning, USA).

\section{Western blot}

Total protein was extracted from CC tissues and cells using the RIPA (radioimmunoprecipitation assay) lysis buffer (Beyotime, China), and the protein concentration was measured by the BCA protein assay kit (Beyotime, China). 25 $\mu$ g protein samples were separated by SDS-PAGE gels, and transferred to PVDF membrane (Thermo Fisher Scientific). After blockage with $5 \%$ skimmed milk at room temperature for $2 \mathrm{~h}$, the PVDF was incubated with the primary antibody against E7 (1:800, Invitrogen, USA), ZNF592 (1:500, Abcam, USA), HMGB1 (1:1000, Abcam, USA), or GAPDH (1:2000, Santa Cruz biotechnology, USA) at $4^{\circ} \mathrm{C}$ overnight. Then, the membranes were washed by PBS three times and incubated with the secondary antibody (anti-rabbit, 1:10000, Thermo Fisher Scientific, USA) for $1 \mathrm{~h}$ at room temperature. After washing 3 times using PBST, the membranes were photographed by the ECL detection system. ImageJ software was used to analyze the western blot bands.

\section{Statistical analysis}

All analyses were performed using SPSS 20.0 statistical software. Comparison between two experimental groups was performed by the Student's t-test. ANOVA was used for comparisons of more than two groups. The Kaplan-Meier analysis was used to plot survival curves, and the log-rank test was used to compare the survival curves. Data were presented as the mean $\pm \mathrm{SD}$. $\mathrm{P}$ values $<0.05$ were statistically significant.

\section{Results}

\section{Circ0036602 was upregulated in CC}

To identify novel circRNAs involved in the progression of HPV positive CC, cervical cancer cell lines Caski and Siha were selected to construct stable transgenic cell lines with E7 oncoprotein knockdown (Caski-shRNA-E7, Siha-shRNA-E7). We performed a circRNA expression profiling microarray analysis on the transgenic (Caski-shRNA-E7, Siha-shRNA-E7) and control cell lines (Caski-NC, Siha-NC). 516 circRNAs were found in both microarrays (Figure 1A). The differentially expressed circRNAs were identified based on the selection criteria: | fold change $(F C) \mid \geq 4$ and $p$-values $<0.05$. Kyoto Encyclopedia of Genes and Genomes (KEGG) analysis revealed that differentially expressed circRNAs were significantly enriched in focal adhesion, regulation of migration, ECM-receptor interaction and JAK2/STAT3 signaling pathway (Figure 1B). Gene ontology (GO) analysis of differentially expressed circRNAs showed that regulation of migration, cell cycle, cell proliferation and TGF- $\beta$ signaling pathway were significantly enriched (Figure 1C). The top 3 upregulated and downregulated circRNAs were selected for further analysis (Table 1), and primers for these circRNAs were synthesized. The expression of these circRNAs was measured in 30 paired HPV positive and HPV negative tissue samples. Among them, circ0036602 was the most significantly upregulated differentially expressed circRNA by approximately 4.07-fold (Figure 1D, Figure S1A). Therefore, circ0036602 was chosen for further analysis. Subsequently, we analyzed the correlation between the expression of circ0036602 and clinicopathological features. The results indicated that circ0036602 expression was positively correlated with degree of differentiation ( $\mathrm{p}=0.02$, Figure 1E), FIGO stage $(p=0.013$, Figure $1 F)$ and $N$ stage $(p=0.017$, Figure 1G). Moreover, high circ0036602 expression resulted in shorter patient's survival $(p=0.01$, Figure $1 \mathrm{H})$. In addition, four cervical cancer cell lines and a normal cervical epithelial cell line were used to assess the expression of circ0036602. In line with the results of circ0036602 expression in cancer tissue, circ0036602 was significantly upregulated in HPV-16 positive cells (Caski and Siha) compared to HPV-16 negative (C33A) and normal cells (Hacat) (Figure 1I). Taken together, these data implied that circ0036602 was upregulated and might be involved in CC progression.

Table 1: 3 up-regulated and 3 down-regulated circRNAs were picked up by circRNA expression profiling microarray analysis.

\begin{tabular}{llllll}
\hline cirRNA & \multicolumn{2}{c}{ chromosome circRNA-type $\begin{array}{l}\text { Gene } \\
\text { symbol }\end{array}$} & $\begin{array}{l}\text { Spliced } \\
\text { length (bp) }\end{array}$ & regulation \\
\hline Circ-0011968 & chr5 & exonic & MTF2 & 1064 & up-regulation \\
circ-0005092 & chr14 & exonic & UBE2E2 & 341 & up-regulation \\
circ-0036602 & chr15 & exonic & ZNF592 & 1053 & up-regulation \\
circ-0013306 & chr6 & exonic & CNOT4 & 1719 & down-regulation \\
circ-0008995 & chr1 & exonic & MFF & 588 & down-regulation \\
circ-0008731 & chr1 & exonic & MAP4K4 & 1375 & down-regulation \\
\hline
\end{tabular}

\section{The characterization of circ0036602 in CC}

To further characterize circ0036602, data from the CircBase online database was analyzed. As demonstrated in Figure 2A, circ0036602 was located on chromosome 15q23.3 and consisted of six exons, spanning from exons 5 to 10 in the host ZNF592. Moreover, we designed a divergent primer and found that circ0036602 could only be amplified in complementary DNA (cDNA) rather than in genomic DNA (gDNA) (Figure 2B). Then, we found that circ0036602 was resistant to digestion by Rnase R, 
while linear ZNF592 was almost completely degraded (Figure 2C), confirming the circular structure of circ0036602. Additionally, to explore the subcellular location of circ0036602, the nuclear fraction was isolated from the cytoplasmic. qRT-PCR showed that circ0036602 was mainly distributed in the cell cytoplasm (Figure 2D). Moreover, FISH staining confirmed that circ0036602 was predominantly located in the cytoplasm (Figure 2E).

\section{Silencing circ0036602 suppressed CC invasion, migration and proliferation}

To explore the functional role of circ0036602 in CC cells, we designed siRNAs (siRNA1, siRNA2) specifically targeting circ0036602 and transfected into Caski cells to silence circ0036602. qRT-PCR results demonstrated that both siRNAs could decrease circ0036602 expression, as shown in Figure 3A. Subsequently, we performed CCK8 assays to investigate the effect of silencing circ0036602 on CC cell proliferation. As shown in Figure 3B, the proliferation ability of Caski cells was found to be impeded after circ0036602 silencing. Wound healing assays demonstrated that knockdown of circ0036602 significantly restrained cell migration in Caski (Figure 3C). Additionally, to further clarify whether circ0036602 affects cell migration and invasion, transwell migration and invasion assays also confirmed that the migration and invasion ability of Caski cells were inhibited after circ0036602 depletion (Figure 3D). Together, these results indicated that silencing circ0036602 inhibited migration, invasion, and proliferation of CC cells.
A

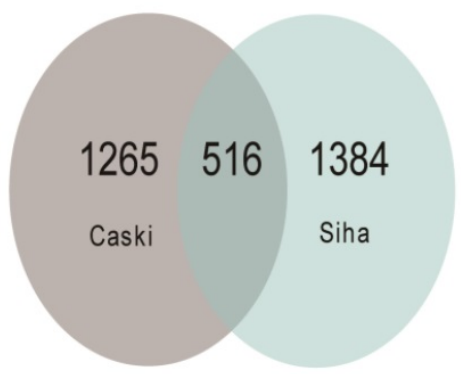

D

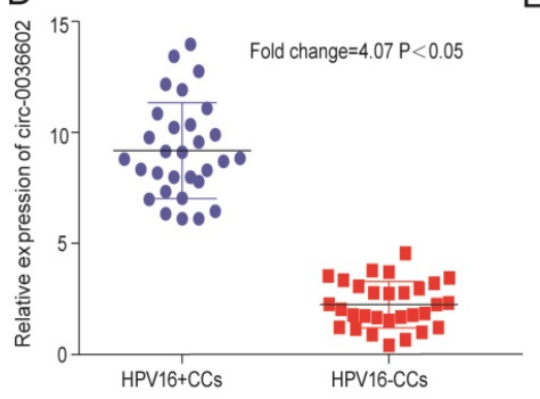

G

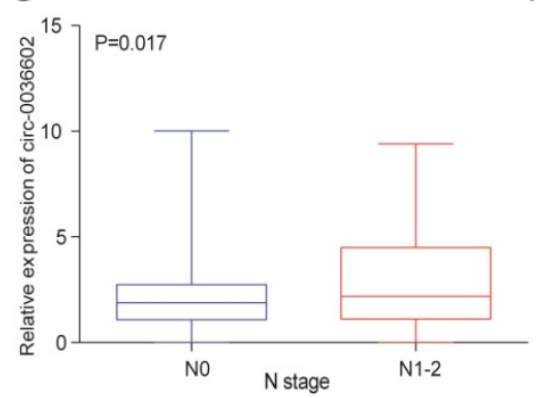

B

E
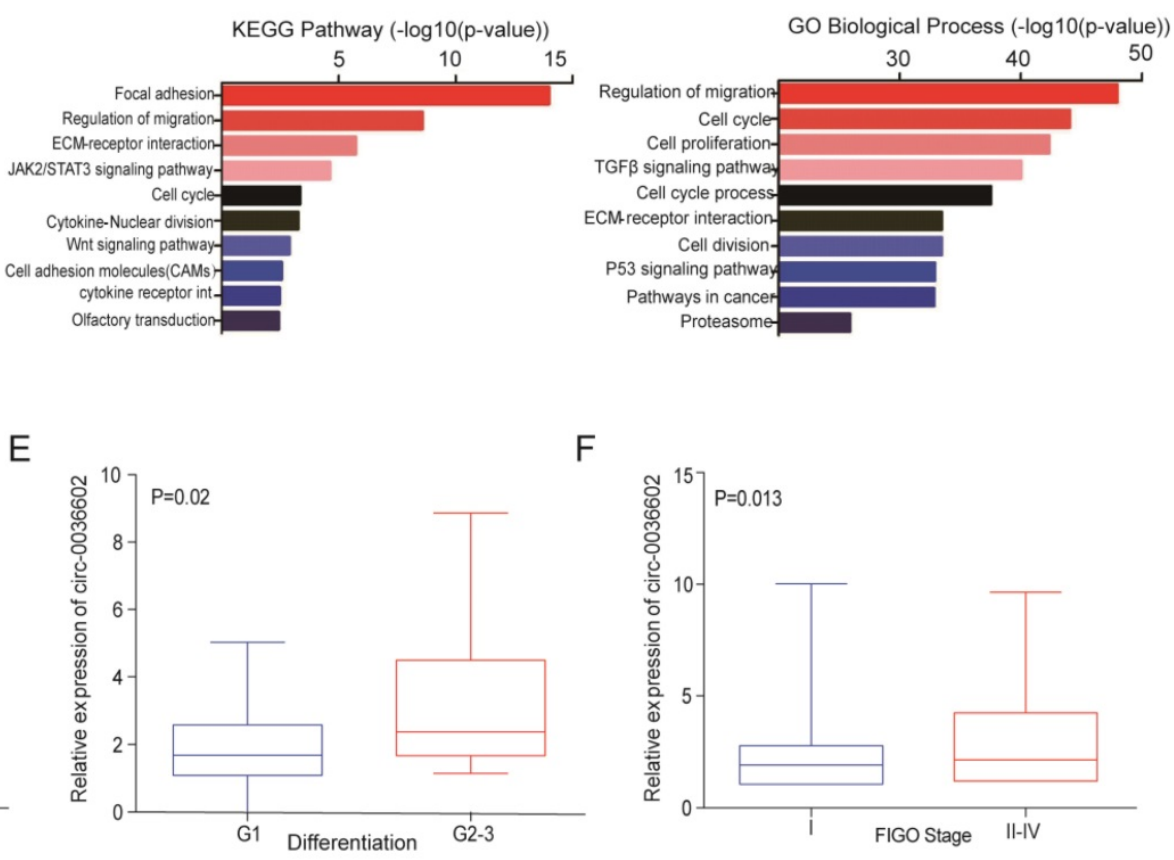

F

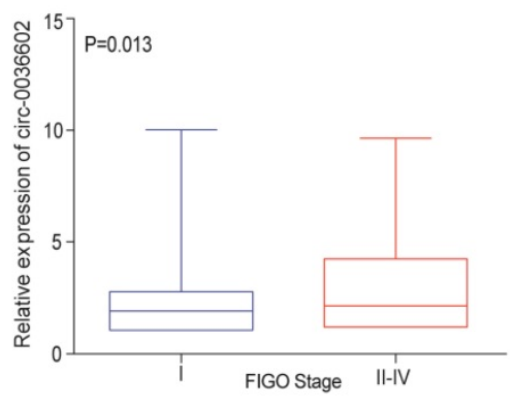

H

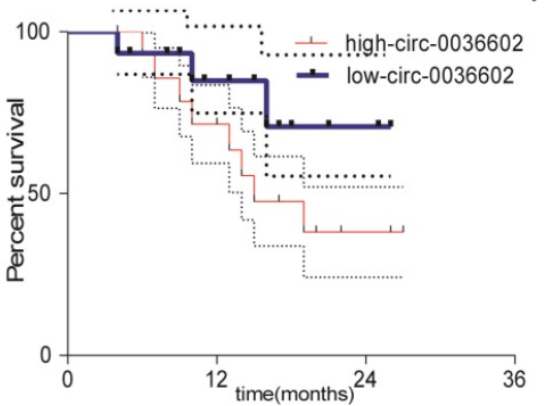

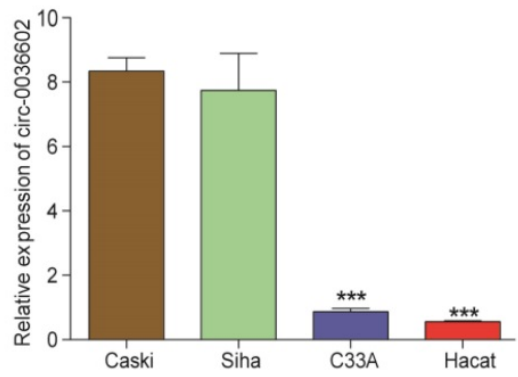

Figure 1: Circ0036602 was upregulated in CC. (A) A total of 516 differentially expressed cirRNAs in Caski-shRNA-E7, Siha-shRNA-E7, and negative control were identified via cirRNAs RNA sequencing. (B-C) Kyoto Encyclopedia of Gene and Genome pathway and Gene Ontology analysis of differentially expressed genes in cervical cancer. (D) The relative expression of circ0036602 in HPV16 positive and HPV negative CC tissue samples. (E-G) The correlated analysis between the relative expression of circ0036602 and differentiation, FIGO stage, and N stage. $(\mathrm{H})$ Kaplan-Meier curves of overall survival of $30 \mathrm{CC}$ patients with low or high circ0036602 expression. (I) The relative expression of circ0036602 in CC cells performed by $q R T-P C R$. * $<<0.05$, Values represent mean \pm SD, $n=3$ independent experiments. 
A

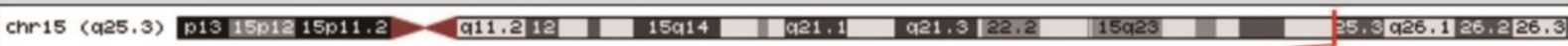

\begin{tabular}{|c|c|c|c|c|c|c|c|}
\hline $\begin{array}{c}\text { Scale } \\
\text { chr15: I }\end{array}$ & $85,335,000 \mid$ & $85,336,0001$ & $85,337,0001$ & 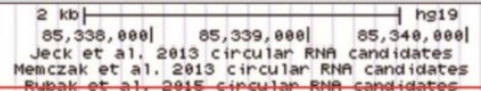 & $85,341,0001$ & $85,342,8081$ & 85,343, (2)e日 \\
\hline
\end{tabular}

Exon5

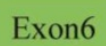

Exon7

Exon8

Exon9

Exon10

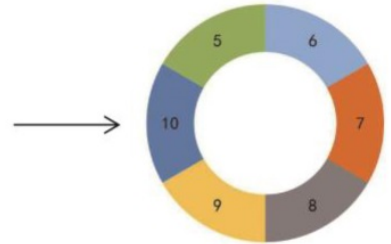

B

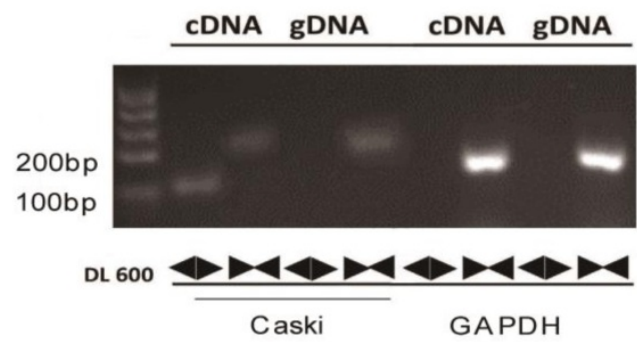

C

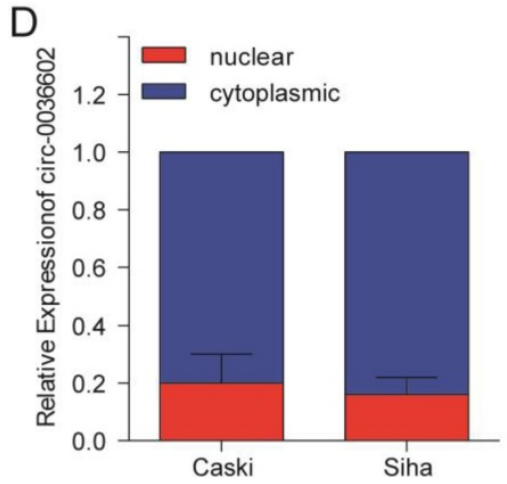

E

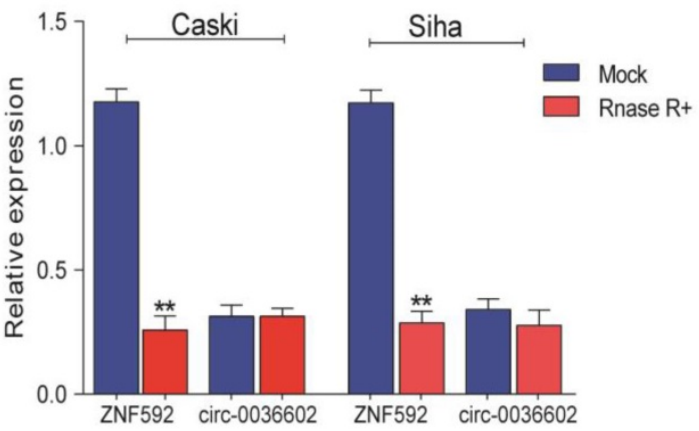

Figure 2: The characterization of circ0036602 in CC. (A) The schematic illustration showed that exons 5 10 of ZNF592 constitute circ0036602. (B) The presence and circular form of circ0036602 established by agarose gel electrophoresis. GAPDH acts as the linear control. (C) circ0036602 and ZNF592 mRNA expression in Caski and Siha cells treated with or without R detected by qRT-PCR analysis. (D-E) The nuclear and cytoplasmic distribution of circ0036602 assessed by qRT-PCR and FISH assay in. ${ }^{*} \mathrm{p}<0.05$, Values represent mean $\pm \mathrm{SD}, \mathrm{n}=3$ independent experiments.

\section{Circ0036602 acted as a sponge of miR-34a-5p and miR-431-5p in CC}

Numerous studies have confirmed that circRNAs, which are mainly localized in the cytoplasm, can interact with miRNAs to regulate the expression of downstream target genes [22, 23]. We thus searched for potential miRNAs associated with circ0036602 using the TargetScan, PITA, and Miranda databases. In total, 5 miRNAs were identified as putative targets, including miR-15b-5p, miR-34a-5p, miR-429, miR-183-5p and miR-431-5p. To further assess whether circ0036602 could bind to these
miRNAs, we performed dual-luciferase assays (Figure 4A). CC cells transfected with miR-34a-5p or miR-431-5p mimics significantly exhibited decreased luciferase activities than the negative control. It has been established that miRNAs can combine with Ago2 to form an RNA-induced silencing complex (RISC) to silence gene expression. Accordingly, the RNA immunoprecipitation (RIP) assay showed that anti-Ago2 suppressed circ0036602 expression (Figure 4B). Therefore, we speculated that circ0036602 might function through competitive binding to miR-34a-5p and miR-431-5p. Previous studies have shown that miR-34a-5p and miR-431-5p were potential oncogenes 
and were downregulated in various solid tumors, including CC, hepatocellular and colorectal cancer [24-26]. To confirm the expression of both miRNAs in CC, qRT-PCR was performed, and the results indicated that both miRNAs were significantly downregulated in HPV-16 positive CC tissues compared with HPV-16 negative ones (Figure 4C and $4 \mathrm{D})$. Moreover, the expression of circ0036602 was negatively correlated with miR-34a-5p and miR-431-5p in CC tissues (Figure 4E and 4F). The above results indicated that circ 0036602 could serve as a sponge of miR-34a-5p and miR-431-5p to regulate their expression negatively.

\section{HMGB 1 was a target of miR-34a-5p and miR-431-5p}

Previous studies discovered that miRNA could

A

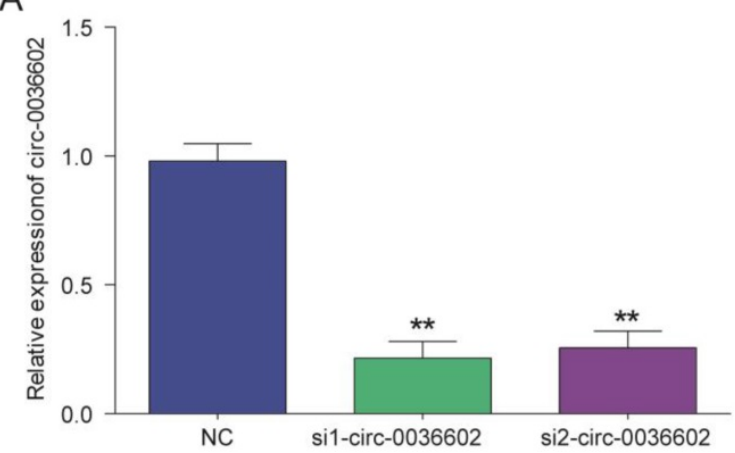

C

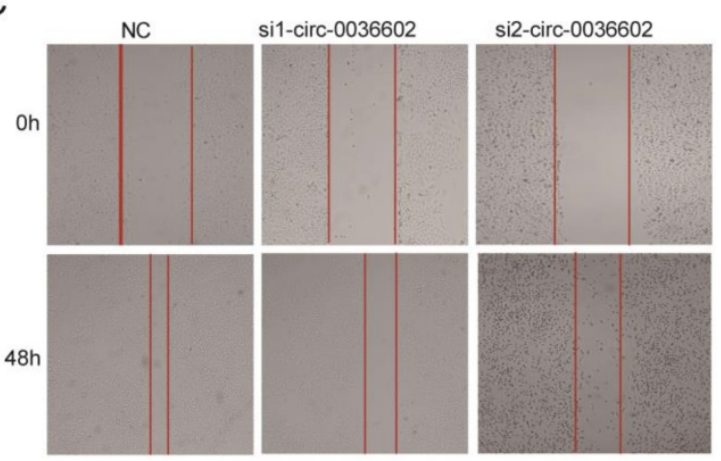

regulate gene expression by binding to $3^{\prime}$-UTR of mRNA [27]. To reveal the potential downstream target genes of both siRNAs mentioned above, CC Caski cells were selected and transfected with si-circ0036602 for RNA sequencing. Cluster analysis demonstrated that 1300 genes were upregulated while 460 were downregulated. Several online prediction tools, including miRase, TargetScan and Miranda were used to identify the miRNA targets. Results showed that 2943 candidates were screened from miR-34a-5p and 1036 from miR-431-5p. The intersection of the candidate genes yielded 10 genes, namely CAMTA1, TP531NP1, MLL5, HMGB1, IRF2BP2, CPSF6, LPHN2, NRXN3, CELF2, and EFNB2 (Figure 5A). Next, we quantified their expression by qRT-PCR and found that HMGB1 was significantly downregulated in Caski and Siha cells
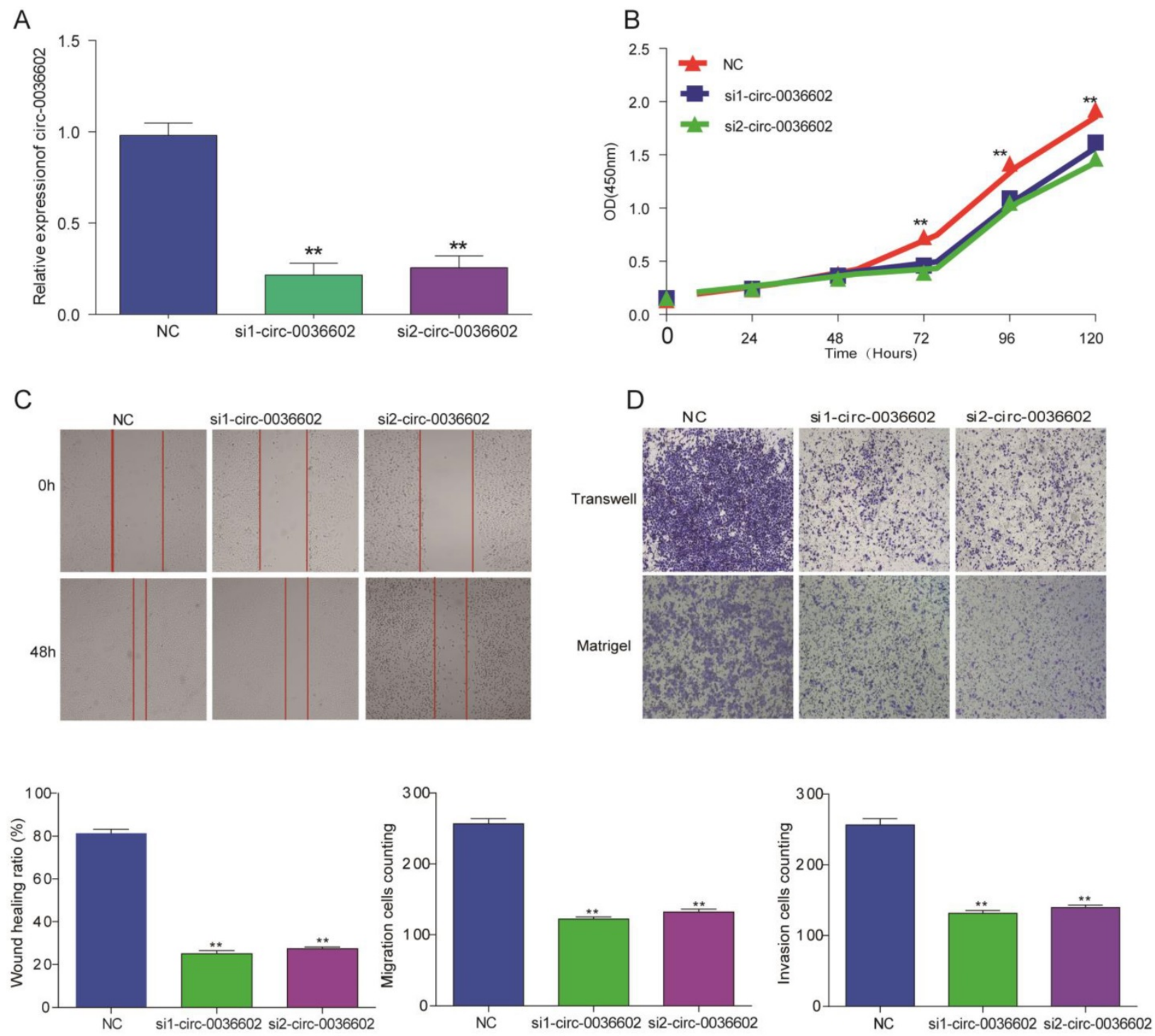

Figure 3: Silencing circ0036602 suppressed CC invasion, migration, and proliferation. (A) The relative expression of circ0036602 in Caski cells transfected with sil-circ0036602, si2-circ0036602, or siNC examined by qRT-PCR. (B) CCK-8 assays were performed to examine the cell proliferation rate at $0,24,48,72$ and $96 \mathrm{~h}$. (C) Wound healing assays showed decreased cell migration ability of Caski cells. (D) Transwell migration and invasion assay confirmed knockdown circ0036602 inhibited the migration and invasion ability. ${ }^{*} \mathrm{p}<0.05$, Values represent mean $\pm \mathrm{SD}, \mathrm{n}=3$ independent experiments. 
(Figure 5B and 5C). Consistently, western blot analysis showed that HMGB1 was downregulated following knockdown of circ0036602 (Figure 5D and 5E). Meanwhile, we found a significant positive correlation between HMGB1 and circ0036602 (Figure
5F) and a negative correlation between HMGB1 with miR-34a-5p (Figure 5G) and miR-431-5p (Figure 5H), which demonstrated that HMGB1 is a target of miR-34a-5p and miR-431-5p, and indirectly regulated by circ0036602.
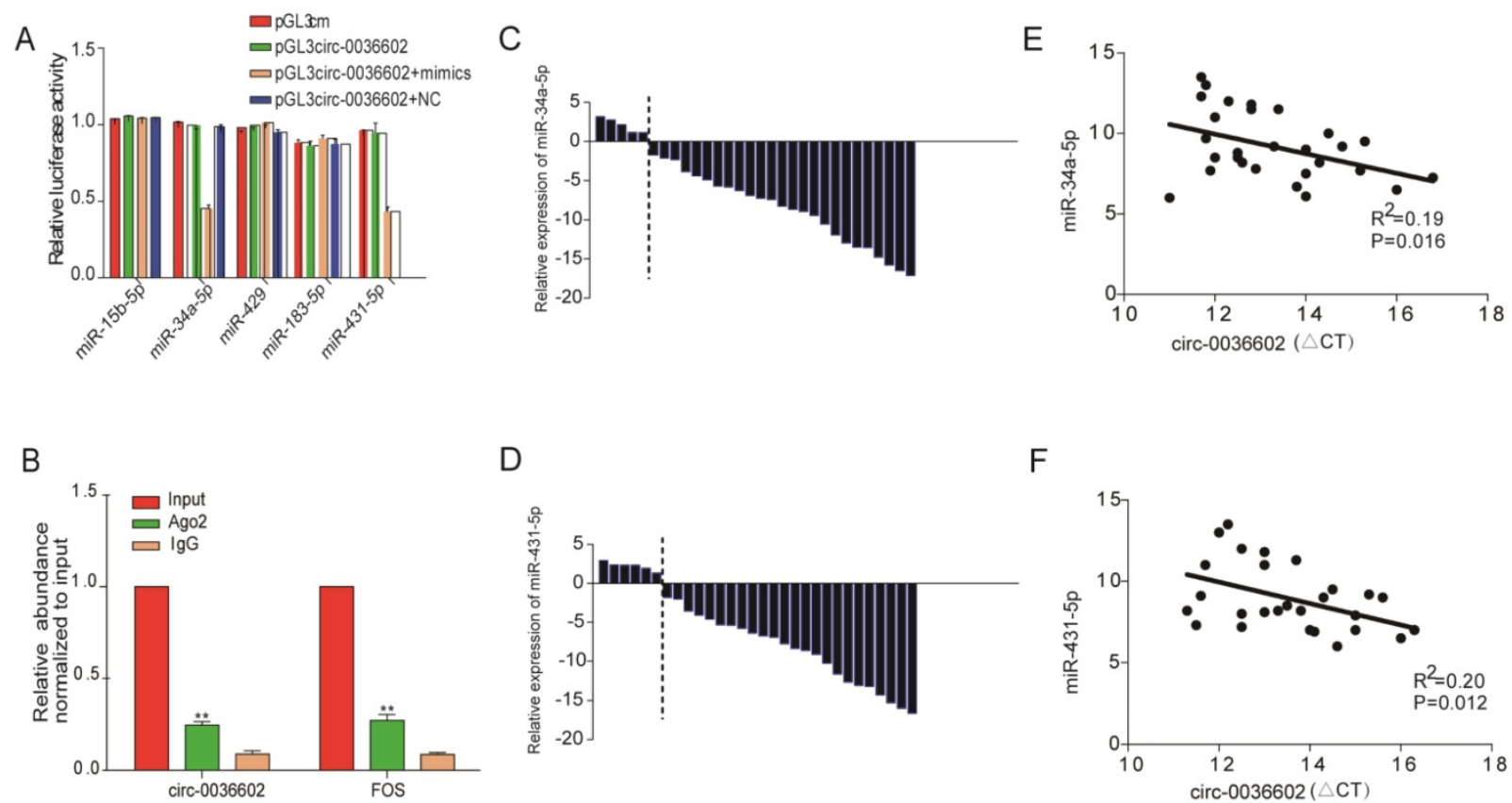

Figure 4: Circ0036602 acted as a sponge of miR-34a-5p and miR-431-5p in CC. (A) Luciferase reporter gene assays confirmed that miR-34a-5p and miR-431-5p can bind with circ0036602. (B) Ago2 RIP experiment showed the combination of Ago2 protein with circ0036602. (C-D) Relative experssion of miR-34a-5p and miR-431-5p in CC and control tissues by qRT-PCR. (E-F) Correlation between circ0036602 and miR-34a-5p and miR-431-5p in CC tissues. ${ }^{*} p<0.05$, Values represent mean $\pm \mathrm{SD}, \mathrm{n}=3$ independent experiments.
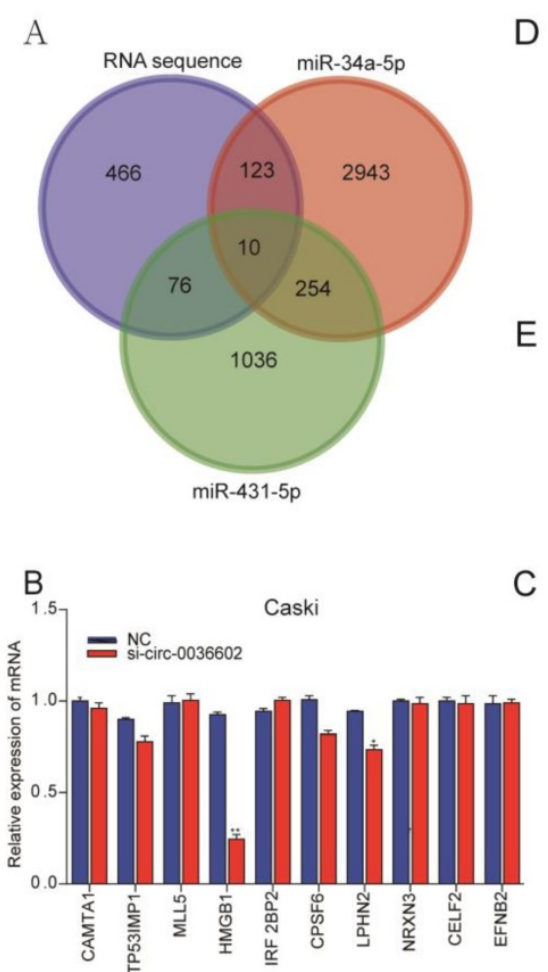

C
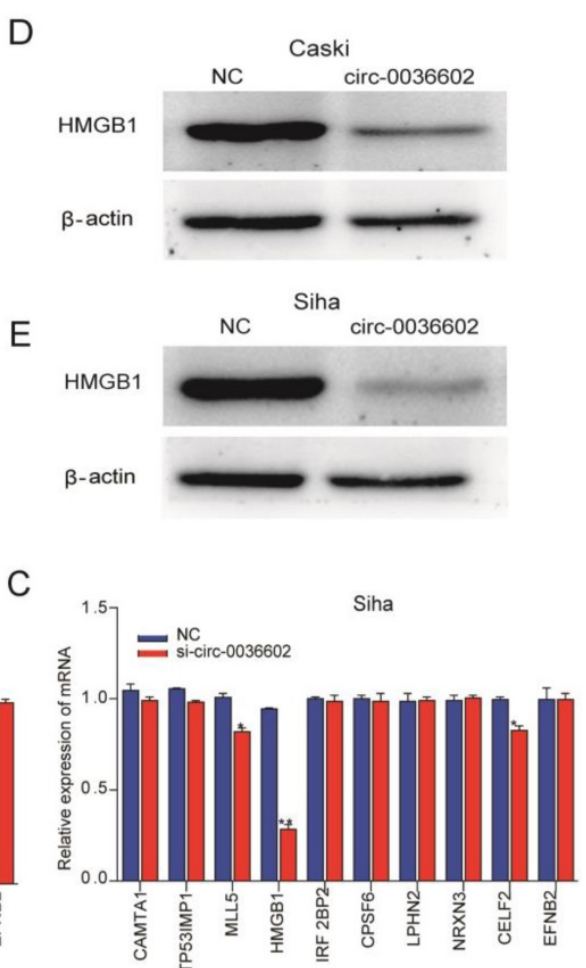
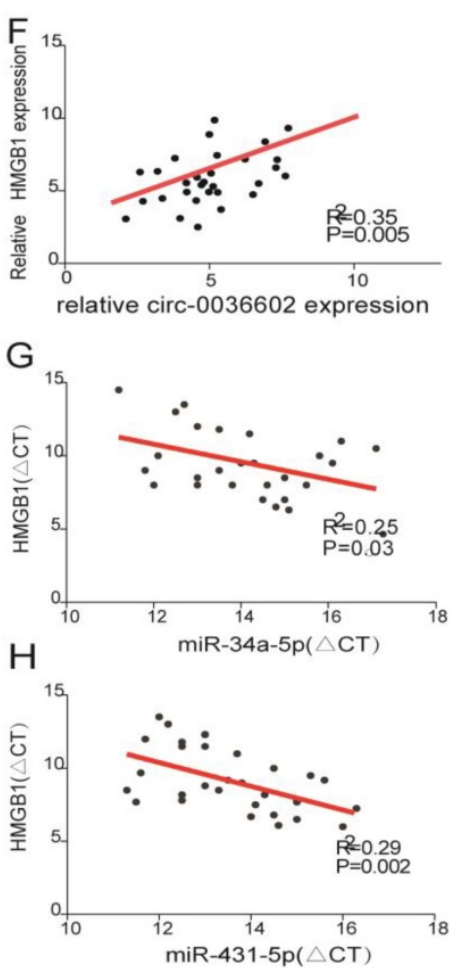

Figure 5: HMGB1 was a target of miR-34a-5p and miR-431-5p. (A) Venn diagram demonstrated the 10 genes from miRbase, TargetScan, Miranda, and RNA sequencing. (B-C) The relative expression of 10 genes in Caski and Siha cells detected by qRT-PCR. (D-E) Western blot verified the expression of HMGB1 in Caski and Siha cells. (F) Correlation analysis between circ0036602 and HMGB1. (G-H) The negative intersection with miR-34a-5p and miR-431-5p and HMGB1. * $<<0.05$, Values represent mean $\pm \mathrm{SD}, \mathrm{n}=3$ independent experiments. 


\section{Discussion}

Discovering novel diagnostic and prognostic biomarkers and unraveling the pathogenesis underlying HPV-16 positive CC is essential to refine current CC treatment efficacy. Numerous circRNAs have been identified in recent years, and their aberrant expression in various cancer have attracted significant attention. Furthermore, circRNAs have been documented as new diagnostic biomarkers and therapeutic targets for cancers [28, 29]. Many circRNAs contain multiple miRNA binding sites and competitively bind with miRNA to mediate its activity [30]. Notwithstanding that several circRNAs have been reported to participate in CC development $[31,32]$, the role of circ0036602 in HPV16-positive CC remains unclear. To the best of our knowledge, this is the first study to investigate the association between circ0036602 and CC and explore the molecular mechanisms of migration and invasion in HPV-16 positive CC. In the current study, we performed RNA sequencing on E7-knockdown CC cells and identified six circRNAs. Finally, we found that a new circRNA generated from the ZNF592 gene was significantly elevated in HPV-16 positive CC tissues relative to HPV16 negative ones. Moreover, circ0036602 was upregulated in HPV-16 positive CC cells, and high circ0036602 expression was negatively correlated with the degree of differentiation, $\mathrm{N}$ stage, and patient survival, suggesting the involvement of circ0036602 in CC. Besides, functional assays indicated that circ0036602 could promote growth and stimulate migration and invasion.

Subsequently, the dual-luciferase reporter and RIP assays showed that miR-34a-5p and miR-431-5p could bind circ0036602 in CC cells. The function of miR-34a-5p or miR-431-5p has been documented in the literature. Wang et al. demonstrated that miR-34a-5p overexpression could inhibit cell viability, migration, invasion and promote apoptosis via Bcl-2 downregulation in cervical cancer [24]. Moreover, Gao et al. showed that miR-34a-5p expression was lower in patients with colorectal cancer recurrence than without recurrence and could inhibit metastasis and recurrence in a p53-dependent manner [25]. Sun et al. identified miR-431-5p as a tumor-suppressor miRNA that negatively regulated ZEB1. By targeting ZEB1, miR-431-5p could inhibit the epithelial-mesenchymal transition induced by ZEB1 [26]. Concordant with the literature, our study showed that miR-34a-5p was downregulated in CC samples and exerted antitumor effects. However, to the best of our knowledge, no studies have documented the regulatory role of miR-431-5p. To identify the target genes of both miRNAs, bioinformatics analysis and RNA sequencing were used, and HMGB1 was identified. Western Blot and qRT-PCR revealed that HMGB1 was upregulated and positively correlated with circ0036602, indicating that HMGB1 is a potential target of miR-34a-5p and miR-431-5p.

HMGB1 is one of the highly conserved chromatin-associated proteins containing HMG-box domains and has been reported to be abnormally expressed in diverse cancers [33]. Chen et al. reported that HMGB1 played a novel role in regulating the Hippo pathway and promoted liver tumorigenesis in an HMGB1-YAP dependent pathway [34]. Furthermore, Gao et al. found that HMGB1 acted as the downstream gene of lncRNAZEB2/miR-204/ HMGB1 to promote pancreatic cancer cell growth and invasion [34]. Moreover, $\mathrm{Li}$ et al. indicated that HMGB1 was upregulated and can be an independent predictor and prognostic biomarker in cervical cancer, consistent with our study findings [35]. In the present study, RNA sequencing and online database analysis showed that HMGB1 is a potential target of miR-34a-5p and miR-431-5p. Moreover, we observed that HMGB1 expression was inversely correlated with both miRNAs, and positively correlated with circ0036602, supporting the hypothesis that HMGB1 is its downstream target. However, further studies are required to substantiate the regulatory role of circ0036602 in CC. In addition, it is conceivable that other circRNAs are involved in the progression of CC, warranting the need for further studies.

\section{Conclusion}

Taken together, this is the first study to document the oncogenic function of circ0036602 in CC. In this regard, circ0036602 blocked the migration, invasion, and proliferation of CC by HMGB1 upregulation mediated by miR-34a-5p and miR-431-5p. These findings revealed that circ0036602 has huge prospects as a therapeutic target and diagnostic biomarker for CC.

\section{Abbreviations}

CC: Cervical Cancer; qRT-PCR: quantitative real-time PCR; CCK8: Cell Counting Kit-8 assay; GO: gene ontology; KEGG: Kyoto Encyclopedia of Genes and Genomes; ATCC: American Type Culture Collection; RPMI: Roswell Park Memorial Institute; FBS: fetal bovine serum; RIP: RNA Binding Protein Immunoprecipitation.

\section{Supplementary Material}

Supplementary figure.

https://www.jgenomics.com/v10p0016s1.pdf 


\section{Acknowledgments}

\section{Funding}

This work was supported by the National Natural Science Foundation of China (8180101591)

\section{Ethics approval and consent to participate}

The authors are accountable for all aspects of the work in ensuring that questions related to the accuracy or integrity of any part of the work are appropriately investigated and resolved. All participants signed written informed consent and the procedure was approved by the Ethics Committee of The Affiliated Cancer Hospital of Nanjing Medical University. All procedures used in this research met the ethical standards set out in the Helsinki Declaration.

\section{Author Contributions}

(I) Conception and design: Y Wu, J Huang; (II) Administrative support: $\mathrm{Y}$ Wu; (III) Provision of study materials or patients: $X$ Ye, J Han; (IV) Collection and assembly of data: B Zhu, X Ye; (V) Data analysis and interpretation: Y Wu, B Zhu, J Han; (VI) Manuscript writing: All authors; (VII) Final approval of manuscript: All authors.

\section{Competing Interests}

All authors have completed the ICMJE uniform disclosure form. The authors have no conflicts of interest to declare.

\section{References}

1. Bray F, Ferlay J, Soerjomataram I, Siegel RL, Torre LA, Jemal A. Global cancer statistics 2018: GLOBOCAN estimates of incidence and mortality worldwide for 36 cancers in 185 countries. CA: A Cancer Journal for Clinicians. 2018; 68(6).

2. Ginsburg O, Bray F, Coleman MP, Vanderpuye V, Conteh L. The global burden of women's cancers: a grand challenge in global health. Lancet. 2016.

3. Human papillomavirus genotype attribution for HPVs 6, 11, 16, 18, 31, $33,45,52$ and 58 in female anogenital lesions. European Journal of Cancer. 2015; 51(13): 1732-41.

4. Hu Z, Ma D. The precision prevention and therapy of HPV-related cervical cancer: new concepts and clinical implications. Cancer Medicine. 2018; 7(9895).

5. Zhai L, Tumban E. Gardasil-9: A global survey of projected efficacy. Antiviral Research. 2016: 101-9.

6. Schiffman M, Doorbar J, Wentzensen N, De Sanjosé S, Fakhry C, Monk BJ, et al. Carcinogenic human papillomavirus infection. Nat Rev Dis Primers. 2016; 2: 16086.

7. Mirabello L, Yeager M, Yu K, Clifford GM, Xiao Y, Zhu B, et al. HPV16 E7 Genetic Conservation Is Critical to Carcinogenesis. Cell. 2017; 170(6): 1164-74.

8. Chen LL, Yang L. Regulation of circRNA biogenesis. Rna Biology. 2015; 12(4): 381-8.

9. Hentze MW, Preiss T. Circular RNAs: splicing's enigma variations. Embo Journal. 2014; 32(7): 923-5.

10. Guarnerio J, Bezzi M, Jeong J, Paffenholz S, Pandolfi P. Oncogenic Role of Fusion-circRNAs Derived from Cancer-Associated Chromosomal Translocations. Cell. 2016; 165(2): 289-302.

11. Jeck WR, Sorrentino JA, Wang K, Slevin MK, Burd CE, Liu J, et al. Circular RNAs are abundant, conserved, and associated with ALU repeats. Rna. 2013; 19(2): 141-57.
12. Cocquerelle C, Mascrez B, Hétuin D, Bailleul B. Mis-splicing yields circular RNA molecules. Faseb Journal. 1993; 7(1): 155-60.

13. Hansen TB, Jensen TI, Clausen BH, Bramsen JB, Finsen B, Damgaard CK, et al. Natural RNA circles function as efficient microRNA sponges. Nature. 2013; 495(7441): 384-8.

14. Zhaoyong, Li, Chuan, Huang, Chun, Bao, et al. Exon-intron circular RNAs regulate transcription in the nucleus. Nature Structural \& Molecular Biology. 2015; 22(3): 256-64.

15. Du WW, Yang W, Liu E, Yang Z, Yang BB. Foxo3 circular RNA retards cell cycle progression via forming ternary complexes with p21 and CDK2. Nucleic Acids Research. 2016; 44(6): gkw027.

16. Yin $\mathrm{Y}$, Long J, He Q, Li Y, Liao Y, He P, et al. Emerging roles of circRNA in formation and progression of cancer. Journal of Cancer. 2019; 10(21).

17. Kristensen LS, Hansen TB, Ven MT, Kjems J. Circular RNAs in cancer: opportunities and challenges in the field. Oncogene. 2018.

18. Zheng Q, Bao C, Guo W, Li S, Chen J, Chen B, et al. Circular RNA profiling reveals an abundant circHIPK3 that regulates cell growth by sponging multiple miRNAs. Nature Communications. 2016; 7: 11215

19. Zeng W, Liu Y, Li WI, Li Y, Zhu J. CircFNDC3B sequestrates miR7 to derepress TIMP3 and inhibit colorectal cancer progression. Molecular Oncology. 2020.

20. Ma N, Li X, Wei H, Zhang H, Zhang S. Circular RNA circNFATC3 acts as a miR-9-5p sponge to promote cervical cancer development by upregulating SDC2. Cellular Oncology. 2020: 1-15.

21. Hu C, Wang Y, Li A, Zhang J, Zhu L. Overexpressed circ_0067934 acts as an oncogene to facilitate cervical cancer progression via the miR545/EIF3C axis. Journal of Cellular Physiology. 2019; 234(5).

22. Chen, Ling-Ling. The biogenesis and emerging roles of circular RNAs. Nat Rev Mol Cell Biol. 2016.

23. Meng S, Zhou H, Feng Z, Xu Z, Tang Y, Li P, Wu M. CircRNA: functions and properties of a novel potential biomarker for cancer. Molecular Cancer. 2017; 16(1)

24. Xiufen W, Yucui X, Jing W. Overexpression of MicroRNA-34a-5p Inhibits suppProliferation and Promotes Apoptosis of Human Cervical Cancer Cells by Downregulation of Bcl-2. Oncology Research. 2017; 26(6): 977-85

25. Gao J, Li N, Dong Y, Li S, Xu L, Li X, et al. miR-34a-5p suppresses colorectal cancer metastasis and predicts recurrence in patients with stage II/III colorectal cancer. Oncogene. 2015.

26. Kexin, Sun, Tiancai, Zeng, Dong, Huang, et al. MicroRNA-431 inhibits migration and invasion of hepatocellular carcinoma cells by targeting the ZEB1-mediated epithelial-mensenchymal transition. FEBS Open Bio. 2015; 5(1): 900-7.

27. Bartel DP. MicroRNAs. Cell. 2004; 116(2): 0-297.

28. Ma HB, Yao YN, Yu JJ, Chen XX, Li HF. Extensive profiling of circular RNAs and the potential regulatory role of circRNA-000284 in cell proliferation and invasion of cervical cancer via sponging miR-506. Am J Transl Res. 2018; 10(2): 592-604.

29. Zhang HD, Jiang LH, Sun DW, Hou JC, Ji ZL. CircRNA: a novel type of biomarker for cancer. Breast Cancer. 2018; 25(1): 1-7.

30. Apratim, Mitra, Karl, Pfeifer, Ki-Sun, Park. Circular RNAs and competing endogenous RNA (ceRNA) networks. Translational Cancer Research. 2018.

31. Song $\mathrm{T}, \mathrm{Xu} \mathrm{A}$, Zhang $\mathrm{Z}$, Gao $\mathrm{F}$, Zhao $\mathrm{L}$, Chen $\mathrm{X}$, et al. CircRNA hsa_circRNA_101996 increases cervical cancer proliferation and invasion through activating TPX2 expression by restraining miR-8075. Journal of Cellular Physiology. 2019.

32. Ma H, Tian T, Liu X, Xia M, Yu L. Upregulated circ_0005576 facilitates cervical cancer progression via the miR-153/KIF20A axis. Biomedicine \& pharmacotherapy = Biomedecine \& pharmacotherapie. 2019; 118: 109311.

33. Goodwin GH, Johns EW. The isolation and purification of the high mobility group (HMG) nonhistone chromosomal proteins. Methods Cell Biol. 1977; 16(1): 257-67.

34. Chen R, Zhu S, Fan XG, Wang H, Lotze MT, Rd ZH, et al. HMGB1 Controls Liver Cancer Initiation through YAP-dependent Aerobic Glycolysis. Hepatology. 2017; 13(1): e0190981.

35. Pan, Li, Mengfei, Xu, Hongbing, Cai, et al. The effect of HMGB1 on the clinicopathological and prognostic features of cervical cancer. Bioscience Reports. 2019. 